\title{
Interpretation: signs and meaning, diversity in language use, equivalences and cultural untranslatability
}

\author{
Jefwa G. Mweri
}

\begin{abstract}
Interpretation, which is an interactive face-to- face communicative event and interpreter's role is active and governed by social and linguistic knowledge of the entire communicative situation - this involves not only linguistic and cultural competence but also appropriate ways of speaking and managing the intercultural event of interpreting. Roy (2000)

This paper examines the practice of interpretation in which interpreters are expected to "successfully make the linguistic and cultural adjustments necessary to convey meaning accurately" Davis (2000). Since interpretation is an interactive face-to face communicative event, how successful can interpreters make such adjustments and therefore convey meaning accurately in Sign Language interpretation?

Effective interpretation is a complex issue. This complexity may result from the fact that "...translators build bridges not only between languages but between differences of two cultures.... Each language is a way of seeing and reflecting the delicate nuances of cultural perceptions, and it is the translator who not only reconstructs the equivalences of the words across linguistic boundaries but also reflects and transplants the emotional vibration of another culture." Schulte (1995)

In this paper, we examine how cultural diversity may affect interpretation of messages by interpreters. Some of the areas we look at include: What problems are inherent in interpretation given that signs do not mean but people mean? And that culture is responsible for teaching us the symbols and what they represent?;Diversity in language use - how does it affect interpretation? ; Does direct and indirect use of language, social customs and relationships, how people express emotions have any bearing on interpretation?; How does lack of equivalences affect interpretation?
\end{abstract}

Key words: Sign Language, Interpretation, Translation, linguistics, context

\subsection{Introduction}

A Sign Language interpreter can be viewed as any hearing person who has learnt a sign language and acts as a mediator in the language barrier that exists between the Deaf and the hearing given that according to the Wikipedia encyclopedia:

sign language is a language which, instead of using acoustically conveyed sound patterns like spoken language does, uses visually transmitted sign patterns (manual communication, body language and lip patterns) to convey meaning-simultaneously combining hand shapes, orientation and 
movement of the hands, arms or body, and facial expressions to express fluidly a speaker's thoughts. This therefore means that an interpreter must at least be bilingual in a spoken language and a signed language.

An ideal interpreter must not only know sign language but he must also be fluent in it, they must be trained and certified.

The importance of interpreters and interpretation in the lives of the deaf is captured in the World Federation of the Deaf (WFD's) vision 2020 in which it envisions that by the year 2020:

Living conditions are good for Deaf people and nothing hinders

participation. Full participation is enabled because all Deaf

people have full access to interpreting services, and interpreters

are professionally trained and qualified. Governments take

responsibility for financing training programmes and

interpreters' costs (McKee 2006).

Effective interpretation however is a complex affair for the reason that it is an active face- to- face event and is governed by social and linguistic knowledge of the entire communicative situation. It therefore involves both linguistic and cultural competences. This is not an easy task given that each language is a way of seeing and reflecting the delicate nuances of cultural perceptions, and it is the translator who not only reconstructs the equivalences of the words across linguistic boundaries but also reflects and transplants the emotional vibration of another culture." Schulte (1995)

The question we want to ask ourselves therefore is: how does cultural diversity affect interpretation of messages by interpreters? Given that the consumers of interpretation and the interpreter come from two distinct languages and cultures, what are the inherent problems faced by the two given that signs do not mean, but people do? How does diversity in language use affect the interpretation process? And is it possible to achieve translatability in cultural diversity in terms equivalences such as -Vocabulary or lexical equivalence, idiomatic and slang equivalence, grammatical and syntactical equivalence and experiential equivalence? This paper seeks to answer these questions.

\subsection{Signs, Culture \& Meaning}

According to Bonvillian (2003), the primary means of interaction between people is language. Speakers use language to convey thoughts, feelings, intentions and desires to others. The language links interlocutors in a dynamic, reflexive process.

Language in its role of linking the interlocutors makes use of symbols which can either be audio or sound based or visual-based. Spoken language makes use of audio or sound based symbols while sign language and written languages makes use of the visual medium. A sign language interpreter is required to be competent in an audio-based symbol system and a visual-based symbol system in order to be effective. However, it is important to note that language symbols are culturally diverse - a diversity that is reflected both in the nature of symbols themselves e.g. Words or signs but also in the rules of their use.( Samovar et al 2007) 
A word or sign does not mean. Meaning is with people. Meanings are internal (i.e., held inside our heads). Words only bring those meanings to awareness as required. This is the reason as to why a word or sign can elicit many meanings depending on one's background and context of use. Thus since signs or words in themselves have no meaning, we have to abstract the meaning from the mind since meanings are internal. In spoken language, words are based on sounds and until interlocutors are able to connect the signifier (sound) and the signified (object), the sound remains a sound. If that connection is made, the sound then becomes a word with meaning.

In KSL there are multiple meaning signs. That is signs that have several meanings and contextual cues are the ones that can determine what they are used to mean. Given that people normally abstract the meaning of words following their unique backgrounds this may pose a challenge to an interpreter who must do the abstractions involving symbols from two different languages. The process of abstraction of meaning is much easier for people who belong to the same culture given the similar experiences they have gone through. The opposite is also true. That if people are from different cultures the process becomes more complicated. Now doesn't it become more complicated if those involved use totally different symbol systems?

Multiple Meaning Words are words that have several meanings depending upon how they are used in a sentence. Context clues help us figure out which meaning is correct.

Examples of multiple meaning signs in KSL (homophones) include the following signs:

1.

a)

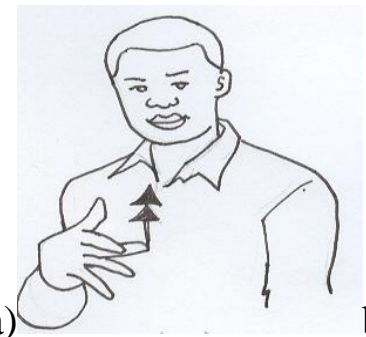

FEEL/EMOTIONS b)

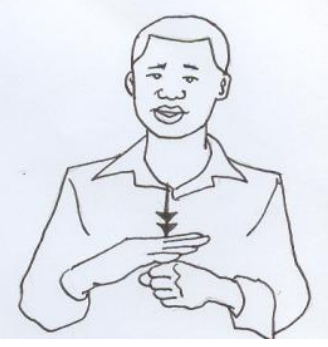

MANY/A LOT c)

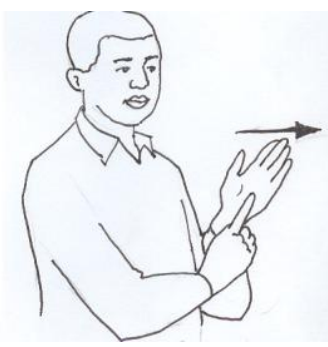

2.

a)

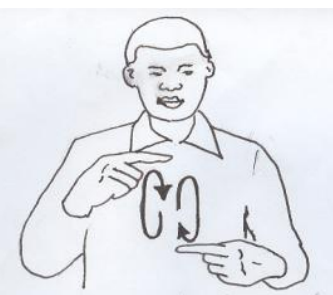

CONTINUE/DEVELOP b)

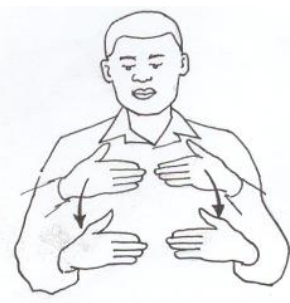

BODY/ PHYSICAL c)

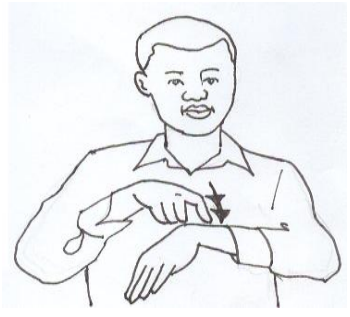

TIME/ WHEN 
3.

a)

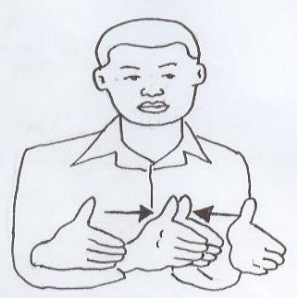

TOGETHER/AND b)

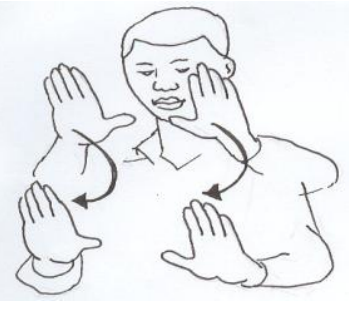

IMPORTANT/KISUMU c)

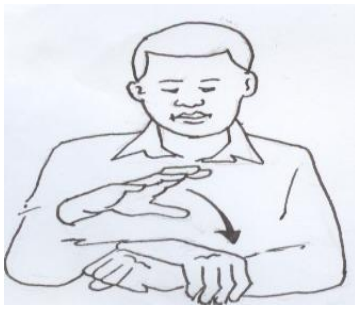

$\mathrm{CATCH} / \mathrm{GET}$

The signs above have more than one meaning. Sign 1a) can mean FEEL or EMOTION; sign 1b) can mean MANY or A LOT: 1c) can have three meaning i.e. FOR EXAMPLE, SHOW or POINT OUT. The signs 2a) - c) also have multiple meanings 2a) means CONTINUE or DEVELOP; 2b) BODY or PHYSICAL; 2c) TIME or WHEN. In 3a) the sign can be used in KSL to mean TOGETHER or AND, while in 3b) the sign can mean IMPORTANT or KISUMU (Kisumu is a Kenyan town). 3c) on the other hand can be used to mean CATCH or GET.

Apart from same signs having different meanings, there are also incidences of same signs with different meanings that may or may not be related. For example the sign for FATHER and MAN and that of MOTHER and WOMAN are the same in KSL. Their articulation is the same but their meanings are different though closely related as compared to the signs above whose meanings are not related.

Let's take the example of the usage of FEEL or EMOTIONS:

4a)

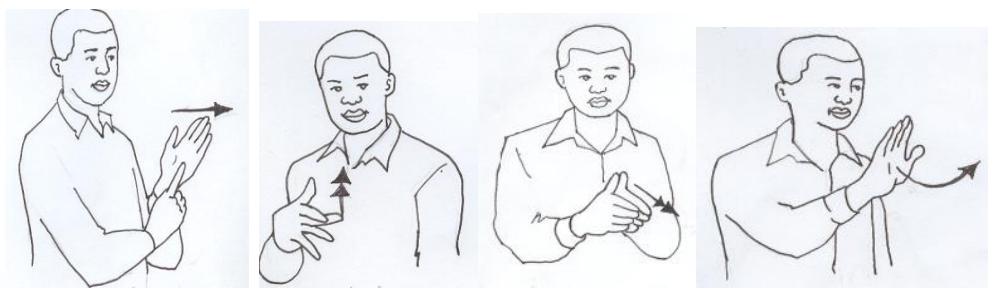

Point out their sexual feelings 
b)

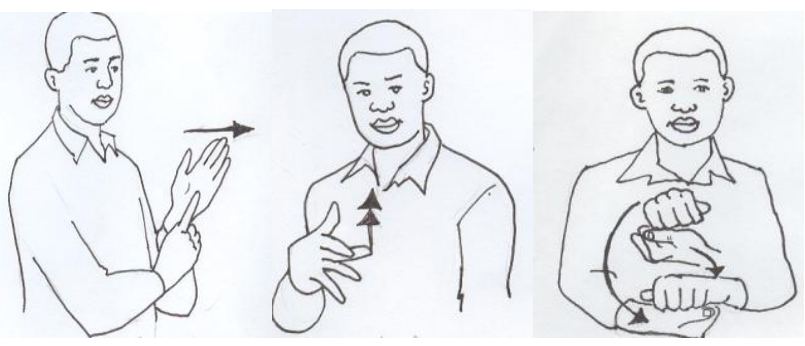

Point out emotional changes

The interpreter in this case must rely on contextual cues to be able to know what the deaf speaker means. Whether it is FEEL or EMOTIONS, the same is true of the signs in the other examples. Another way of discerning meaning by the interpreter is by being able to rely on the mouthing patterns of the signer. Deaf people normally have their signs accompanied by mouthing patterns. According to Valli and Lucas (1995:81) there are three types of mouthing or mouth patterns:

Full mouthing: Here words are pronounced without voice.

Reduce mouthing: the words are not fully pronounced.

Lexicalized mouthing: E.g. the mouth configuration of FINISH and HAVE (ASL) which clearly derive from English pronunciation but have become part of ASL signs.

Mouthing or what Spence and Woll (2005) call the spoken components of SL have various uses:

a) to represent spoken language mouth pattern in combination with signs

b) to represent spoken language mouth patterns with first letter signs, and ;

c) to distinguish other manual homonyms

Deaf Kenyans mostly mouth in English. As Woll (1990:958) asserts signers use mouth patterns as a result of their exposure to spoken language. Mouthing is a phenomenon borne out of contact between a sign language and a spoken language. Mouthing forms the "spoken" components of Sign language. KSL users tend to mouth certain signs that match with words in a spoken language in this case English or Kiswahili. Though English forms the predominant part of mouthing, due to significant KSL-English contact in schools, according to Jefwa (2009), the contact between KSL and Kiswahili though minimal has brought about some Kiswahili words finding their way into KSL, however these words do not enter KSL as words of their own per se but they are used with existing signs thus no new signs are formed to accommodate them. Examples of Kiswahili mouthed signs include:

MZUNGU (A European)

JOGOO (A cockerel)

BASI (That's all)

BADO (Not yet)

SAFARI (Journey)

MIA (One hundred)

HAPA HAPA (Just here)

POA (Fine) 


\section{WEE! (An exclamation for you)}

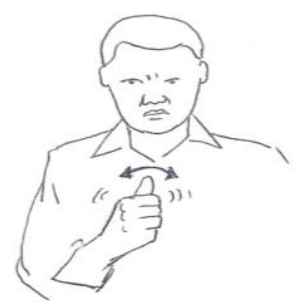

BADO (Not yet)

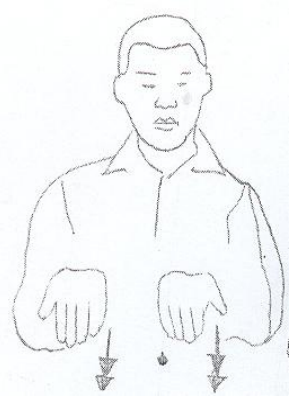

HAPA HAPA (just here)

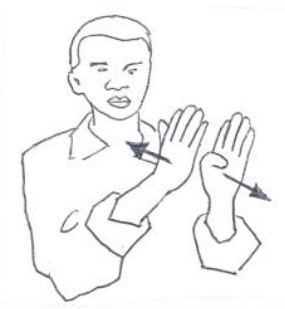

BASI (That's all)

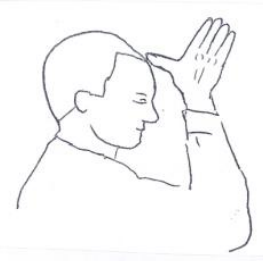

JOGOO (A cockerel)

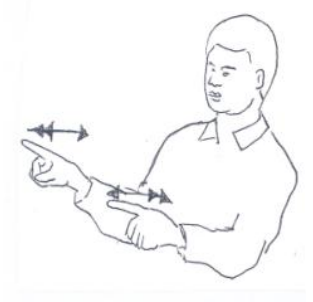

SAFARI (Journey)

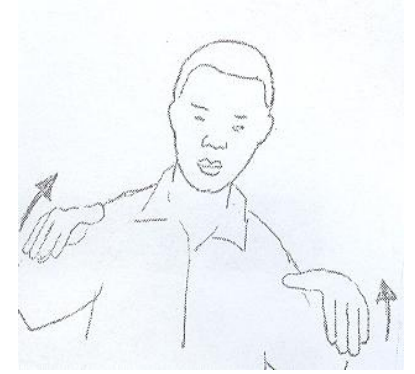

POA (fine)

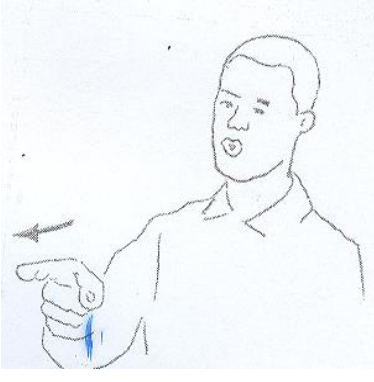

WEE! (YOU!)

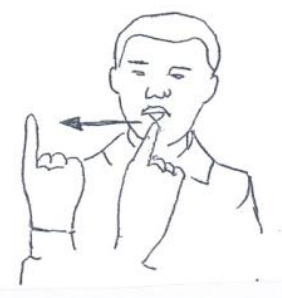

MIA (one hundred)

The Kiswahili mouthed signs on the other hand are used to represent spoken language mouth pattern in combination with signs. These may pose a challenge to an interpreter who is not so conversant with Kiswahili. The interpreter may know the sign e.g. MIA (for one hundred Kenya shillings) but the mouthing may confuse them due to limited Kiswahili Knowledge or since the interpreter is aware that a KSL user will most likely mouth in English, they may anticipate the same and may get confused in the event that the signer uses Kiswahili mouthing.

In the case of English mouthing, the third function of mouthing discussed by Woll above applies where mouthing in this case is to distinguish other manual homonyms that is same signs but different meaning as in FEEL/EMOTIONS; MANY/A LOT; FOR 


\section{EXAMPLE / SHOW/POINT OUT; CONTINUE/DEVELOP; BODY/ PHYSICAL; TIME/ WHEN TOGETHER/AND; IMPORTANT/KISUMU; CATCH/GET.}

While contextual clues may help an interpreter abstract the meaning of homonyms, mouthing may pose a challenge the interpreter since it may be dependent on proximity in terms of the interpreter and the deaf signer bearing in mind that some of the mouthed words may not be articulated at the lips and an interpreter may find it difficult to use mouthing patterns as a way of abstracting meaning in an interpretation situation.

Other examples in KSL include: NAIROBI/MINISTER/BLUE/WHEN; WANT/LIKE; STATUS/CAKE SITUATION; ACTIVITY SPORTS; CAN/YES/POSSIBLE; KISII/HOMOSEXUAL; PREACH/ LECTURE; ACCIDENT/CHALLENGE etc.

Another possible source of difficulty for a KSL interpreter would be the issue of sign variations. There are certain KSL signs that have more than one form. For example apart from the standard KSL signs for RICE and FISH for example there are other variants of the same sign. RICE for example has two variants while fish also has about two variants. Other examples include MOTHER, MANGO, SEX, VAGINA etc. These variations of the standard KSL signs are articulated differently and as a result of regional differences. However these variants cannot impede communication among the deaf signers themselves because they only serve to show that the deaf person comes from a certain region of Kenya. However for an interpreter who may not be aware of these differences it may pose a major problem in terms of relying messages. This is an example of diversity even within cultures that share the same language where there are some regional variations in terms if signing.

\subsection{Diversity in language use}

How we communicate (style) is a product of our cultures and it can lead to misunderstandings because types of communication patterns are related to cultural differences. Diversity in language use may manifest itself in the following ways:

- High involvement patterns VS. High considerateness

- Direct VS. Indirect communication

- Expression of emotional affect

- Maintenance of Social Customs and Relationships

\subsubsection{High involvement patterns VS. High considerateness}

According to Deborah Tannen, diversity in language use is dependent on cultural differences and she divided cultures into high involvement and high considerateness:- 
High involvement cultures exhibit the following characteristics in language use: They talk more, interrupt more, expect to be interrupted, talk loudly, more quickly, and enjoy argument. High considerateness cultures on the other hand exhibit the following characteristics: They speak one at a time, use polite listening sounds, don't interrupt, give respectful responses to their conversational partner. Deaf Kenyans fall under larger dominant culture of the hearing which in terms of communication falls under high considerateness. The deaf too exhibit the characteristics of this style. In an all deaf conversation, they respect each others turns; they hardly ever interrupt and mostly speak when their turn comes.

The visual nature of their communication may largely contribute to this high considerateness in conversational style. As Okombo et al (2006) assert, the significant role played by the tongue in spoken languages is played by the hands in sign language. So, signs have many properties based on the hand, that is manual properties. However, sometime manual properties work hand in hand with non-manual grammatical markers to make meaning. Okombo et al continue to say ... the signer may add more meaning to the signs by movements and positioning of the eyes, eyebrows, mouth, face, head, shoulder and the body. These movements may give the same hand- sign different meanings with regards to matters of asking, confirming or expressing doubt about something. Thus in a communicative situation the deaf has to be very attentive so they don't miss out on meaning that is non-manual based.

For the interpreter too the conversational style adopted by the deaf can also pose major challenges. If the interpreter is for instance from a high involvement culture, they are likely to misunderstand their deaf clients. But the biggest challenge will emanate from the interpreters inability to connect the non manual and manual signs thus communicating wrong messages.

\subsubsection{Direct vs. Indirect use of language}

People who come from cultures that use language directly, especially the Americans, like to get straight to the point, don't beat round the bush, would like to get down to business right away. While people who come from cultures that use language indirectly, talk in around about way, believes in saving face and interpersonal harmony e.g. the Japanese have 15 ways of saying no; they cannot say I don't agree with you, or you are wrong. The direct use of language by Americans stems from the fact they believe that honesty is the best policy and this is reflected in their way of communication.

Kenyans generally are indirect users of language. However the deaf co-culture uses language directly. They go straight to the point and say what they think bluntly without regards to the feelings of the other party. While the dominant culture of the hearing in Kenya uses language indirectly reflecting the cultures propensity for consideration of others, and saving face is crucial, the deaf use language frankly and explicitly. While a hearing person would say I am sick and would not say where (if for example the sickness is related to the private parts), a deaf person will directly refer to the part directly. The direct use of language by the deaf poses a big challenge to an interpreter who most likely comes from a culture that uses language indirectly. Meaning is likely to suffer in this 
regard since the interpreter may not be able to translate certain concepts that may seem "embarrassing".

\subsubsection{Expression of emotional affect}

Similarly, emotional affect is expressed differently depending on one's cultural background. People in some cultures are more reserved than others, some express their feelings more freely and openly than others. The expression of emotional affect may be done through the use of euphemisms that assists the speaker avoid expressing strong feelings such as anger or love. Kenyan deaf people fall under the dominant culture where emotions are not shown publicly. However to most hearing Kenyans, the deaf are highly emotional and they are to be avoided. This view may affect the way an interpreter conveys emotions of the deaf in an interpretation situation. Interpreters will be expected to convey the emotional emphasis of the speaker by mimicking the speaker's emotions through their voices and dramatic gestures.

They must employ all the visual cues contained in the language they are interpreting. However sometimes in certain context an interpreter may not be able to do this for fear of reprisal. A case in point is courtroom interpretation. A story is told of how an interpreter in a court of law in Kenya landed in jail for trying to be accurate in expressing the deaf person's emotions. When the deaf person uses emotive words such as foolish or stupid the judge decided to punish the interpreter for using those words in his court while in actual sense it was the deaf person who did. Kenyan deaf people use direct emotional affect which can be a challenge as illustrated above.

\subsubsection{Maintenance of Social Customs and Relationships}

Cultural diversity in language use may also be reflected in the way each culture maintains social customs and relationships (Samovar et al 2007: 117). Language in some cultures is used to enhance social status and relationships. For example among the Giryama in Kenya, when talking to a person one respects they tend to pluralize the person. For instance, when talking to a father-in-law, A Giryama will say "we are going to the market" instead of "I am going to the market" when referring to himself going to the market alone. This pluralization of person is a sign of respect. This is also true of most Kenyan cultural groups who ultimately become interpreters. The use of language to reflect social status can pose a challenge to an interpreter who may be interpreting for a deaf Kenyan who does not have this feature of language use. Thus the interpreter may keep on referring to "we" instead of "I" that the deaf person is using when the person involved is of high status or needs to be respected thus sending wrong messages.

\subsection{Equivalences in Interpretation}

An interpreter has an ethical responsibility to accurately convey meaning. Interpretation is a complex affair. It is not always easy to accurately interpret from one language to another. Spontaneous interpretations are difficult for many reasons including the fact that:

- Signs are culturally bound and they may not have direct equivalents 
- Cultural orientation can render a direct translation nonsensical

- A culture may not have the background and understanding to translate experiences specific to another culture

- There is no full equivalences in any translation

Bearing the above in mind, an interpreter may experience difficulties securing adequate interpretation as discussed below:

\subsubsection{Vocabulary or lexical equivalence}

In interpretation the sole aim of the interpreter is to convey the meaning and style of the original language. However this sometimes is challenging given that certain lexical items may not have equivalents in other languages, thus the interpreter has to find a solution to this lack of equivalences. For instance most African languages have no single lexical item that stands for uncle or aunt they are just my father's or mother's brother or sister respectively. KSL too does not have equivalences for the same. The lexical items being used are borrowed.

The same is true of other close relations. For example, what would be known as a cousin in English is just a brother or sister in most African languages. Another example will be the word Miss in English as exemplified in "I have missed you." There is no equivalent to the word "miss" in Kiswahili. The nearest will be "nimekukosa." Kosa is the Swahili verb for miss for example in "Juma alikosa mtihani." "Juma missed the exam." This Kiswahili sentence is perfectly correct but "nimekukosa" for I have missed you does not make sense. The nearest to I have missed you in Kiswahili would be I am longing for you which would be "nimekutamani" which does not bring out the same sense as I have missed you.

KSL for example does not have an equivalent for the word Love as used in most western societies. Love in these societies may include parental love- parents loving their children or children saying they love their parents. This kind of love is hardly expressed in most African languages,be KSL included. The love that is likely to expressed is that of "boy loves girl or girl loves boy." Parental love is not expressed openly. The "boy loves girl or girl loves boy." This kind of love is accompanied by non manual markers that would complement the love. The same non-manual markers cannot be used for a girl loving his father or a boy loving his mother. This creates a dilemma for the interpreter when for example interpreting from English to KSL and has to translate the idea of a parent loving her child and again a girl loving a boy. This may mean that when interpreting the parental love, the interpreter may use words that are not in synchrony with the emotions, thus distorting meaning.

Another problem with translation according to Nida (1958) is that there is no one- to- one correspondence between a lexical item and its meaning thus making verbatim translation impossible. This is also true of interpretation which in most cases is spontaneous. This arbitrariness of the linguistic sign makes interpretation difficult. Nida calls this skewing and asserts that in SL it is difficult to find exact synonyms since the lexicon (the 
collection of words used across the language) tend not to match. The question posed here is in spontaneous interpretation, when a signer uses a multiple meaning lexical item which meaning should the interpreter choose? What if the interpreter is not aware of the multiple meanings of the word? Skewing therefore can lead to misrepresentation of information. Prahal \& Petzol (1997) distinguish between translation problems (TP) and translation difficulties (TD). TP they say are known problems e.g. a source term having multiple target translation. TPs are translator-independent. TD on the other hand are issues facing the individual translator during translation. According to Gophinathan (1993), TP can be divided into three problems of meaning resulting from words having:

i) Suggestive meaning as well as literal meaning

ii) Socio- cultural meaning such as culturally specific lexical items, idioms and folk images

iii) False cognates

Words mean but they also imply. It is therefore an interpreter's duty to be able to understand both the meaning and implication of a sign, i.e. the denotation and connotation of the sign. Take the sign HYENA. Its referential meaning in the sense of denotation is based on the factual description of the animal it denotes. However we can use it to refer to human beings who remind us (in some way) of the characteristics (for example, greed) associated with the animal. (Okombo et al 2006: 92).

Though we will deal with socio-cultural meaning will be dealt with in the next section, false cognates can also present a big problem in interpretation. False cognates are pairs of words in the same or different languages that are similar in form and meaning but have different roots. That is, they appear to be or are sometimes considered cognates when in fact they are not. Note that even false cognates may have an indirect connection between them, even if they lack a common root. For example the sign for FATHER in ASL, is the sign for COCKEREL in KSL.

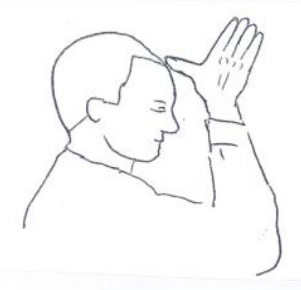




\section{FATHER (ASL)/ COCKEREL (KSL)}

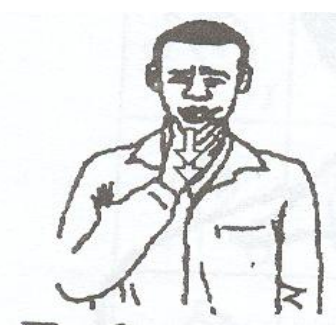

\section{FATHER/MAN/MALE (KSL)}

Other lexical items that can present problems in interpretation include specialized terminologies or jargon. For example, jargon can be words related to the computer: RAM, Hard Disk Drive, CPU, Graphics Card etc. are jargon and may be hard to interpret. According to Arnold et al (1994), specialized terminologies may encounter lexical holes or where a lexical item does not have a lexical equivalent in another language. The examples above of words within the computer field present a clear case of lexical holes since they do not have lexical equivalents in KSL. These terminologies will be hard to interpret unless the translator or intrepreter use loan words, or create new terms or find a cultural substitute.(Beekman and Callow 19997; Saracevic 1989)

\subsubsection{Ideomatic or Slang Equivalence}

Idiomatic expressions are culture bound and present interpretation problems. An idiom is an expression, the meaning of which is not immediately apparent from the words that make up that expression. For example, one common example in English is "kick the bucket," which does not mean that one actually kick a bucket; it mean that someone has died. A KSL interpreter who does not understand this English idiom may literally interpret it to mean that one physically kicked a bucket. Others used in the Kenyan spoken language scene are: beating round the bush- that is not being straight to the point; shaking hands with an old friend- meaning going for a short call; In black American English there is Jumping the broom; If an interpreter is not familiar with these idioms which belong to different cultures then they are likely to misinterpret their meaning. A common one used by deaf Kenyans is one that indicates that one is going to make a phone call to mean going to the toilet. If an interpreter literally talks about the deaf 
person having gone to make a phone call and not gone to the toilet how nonsensical would the interpretation be. There are also slang terms used in KSL for MONEY, SEX, BEAUTIFUL, RUN etc. For one to be able to translate idiomatic expressions and slang terms, they need to be familiar with the idioms and slang terms of that particular culture

\subsubsection{Grammatical- Syntactical Equivalences}

Lack of equivalences in parts of speech can also be problematic in interpretation. For example Kiswahili does not mark for the third person overtly like English does. In English the third person is marked by He/She and It. Though it is neutral and does not mark for gender, she and He mark for gender which Kiswahili does not. The English sentence He went to the market would translate as "A-li-enda sokoni" A- marks the person but does not tell us whether the person who went to the market is male or female. Li- marks tense and - enda is the verb go. If an interpreter was interpreting from Kiswahili to KSL they would find it difficult to know exactly whether the person went to the market was male of female. The same sentence in KSL would also present difficulties since it would be MARKET GO since pronouns in KSL are marked in signing space and the noun must have been referred to prior. After the first mention of a noun the subsequent mentions can be by pointing at the place the noun was placed. The pointing transforms the noun into pronouns. In KSL HE/ SHE and IT are signed on the sides using same manual signs.

\subsubsection{Experiential- Cultural equivalence}

Interpreters may also come across structural and cultural differences between languages. Sechrest et al (1972) indicate that experiential equivalence refers to the fact that in order for translation to be successful from one culture to another, they must utilize terms referring to real things and real experiences which are similar in both cultures if not exactly familiar. Experiential equivalence is also known as cultural translation i.e. an item being translated must have the same cultural meaning in two languages.

Shared experience is crucial for effective interpretation because the meanings cultures have for words or signs are based on shared experiences. Thus for example a KSL interpreter may have difficulties differentiating between ICE and SNOW since it's neither part of the deaf Kenyan experience nor the interpreter's experiences. Similarly, 
for example people whose cultural experiences do not include rivers, streams and no ocean may not have a word for ocean. Thus when they come across an ocean they may refer to it as a river. The interpreter may have some translation difficulties knowing that in this instance River means Ocean.

\subsection{Conclusion}

From the above discussion, we have seen the challenges that an interpreter is likely to encounter given the two distinct symbol systems and cultures. According to Samovar "words, like nature, half reveal and half conceal the soul within." Samovar adds that what is "half concealed may often be more important than what is concealed." Such is the dilemma that an interpreter has to deal with. Multiple meaning signs, Diversity of language use and lack of equivalences at different levels present the interpreter with challenges.

\section{References}

Arnold, D., Bulkan, L., Lee Humphreys, R.L., Meijer, S., and Sadler, L. (1994). Modern

Translation: An introductory guide. Cambridge Mass.: Blackwell

Diekema, R.A. (2003). Translation Events, in Cross- language information retrieval: Lexical Ambiguity, Lexical Holes, Vocabulary Mismatch, and correct Translation. Dissertation. School of information studies. University of Syracuse

Gopinathal, G. (1993). The Nature and Problems of Translation. In; Problems of Translation. G. Gopinathal and S.Kandaswamy (eds.) Allahabad, India: Lokbharati Parakashal: 37-50

Jefwa, G. (2009). Structural borrowing: the case of Kenya Sign Language (KSL) and Kiswahili contact signing. In Iraki, F.K. (Ed.) The Journal of Language, Technology \& Entrepreneurship in Africa. Nairobi: United States International University 
Nida, E.A. (1958) Analysis of meaning and dictionary making. International Journal of American Linguistics. Vol. 24. 27-292.

Napier J., McKee, R., \& Goswell, D. (2006). Sign Language Interpreting: theory and practice in Australia and Newzeland. Sydney: Federation press.

Okombo, O., Akaranga, W., Mweri, J.G. \& Ogutu, T.A.A. (2006). Introduction to the theory and Skills of teaching Kenyan Sign Language: A handbook for teachers. Nairobi: KSDC.

Prahal, B., \& Petzol, S. (1997). Translation Problems and Translation Strategies involved in Human and Machine. Empirical studies. In Machines Translation and Translation theory. C. Havenschild and S. Heizmann (eds.) Newyork NY: Mouton Gruyter.

Roy, C.B. (Ed.). (2000) Innovative Practices for Teaching Sign Language Interpreters. Washington, DC: Gallaudet University Press.

Saracevic, T. (1975). Relevance: a Review of and a frame work for thinking and the notion in information Science. Journal of American society for information services. Vol. 26.

Samovar, L.A., Porter, E.R., \&McDaniel E.R. (2007) Communication between cultures. United States: Thomas Wadsworth

Schultz, J.M. (1991). Mapping and cognitive development in the teaching of foreign language writing. The French Review 64, no.6

Valli, C. \& Lucas, C. (1995). Linguistics of American Sign Language: An introduction. Washington DC: Gallaudet University Press

Woll, B., \& Spence, R.S. (2005). The Linguistics of British Sign Language : An Introduction. London: Cambridge University press 